\section{Cytomegalovirus Ileocolitis: Different Manifestations of the Same Illness in the Terminal Ileum and Colon}

We report here a case of cytomegalovirus ileocolitis in a 38-yearold, HIV-positive man in state C 3 , who was admitted to hospital with chronic diarrhea. The colonoscopy revealed marked signs of inflammation of the mucosa of the colon and terminal ileum. In the distal tracts, areas of petechia, edema, and erythema were prominent. Elsewhere in the colon, including the cecum, there were large, irregular ulcerations, some of which had a wide, craterlike appearance (Figure 1), while others were extremely deep; they all presented a multi-colored appearance. The ileum presented minute aphthoid ulcerations and areas of submucous hemorrhage (Figure 2). Biopsies were taken, which confirmed the diagnosis of cytomegalovirus of ileocolitis when the typical intranuclear inclusion appeared within any histiocytes of lamina propria.

The patient was treated with ganciclovir; the treatment relieved his fever and diarrhea. Five months later, the patient remains asymptomatic, without diarrhea; a control colonoscopy was not performed, because the patient refused it.

The intestinal alteration in the cytomegalovirus infection is located mainly in distal segments of the small bowel and the right colon (1), and usually occurs in immunocompromised patients, such as those who are undergoing bone-marrow transplantation (2), or in AIDS victims (3). In AIDS, there is a pattern of multiple, diffuse and small ulcerations that is largely right-sided, and is produced by cytomegalovirus (4), although neither the degree, type, nor extent of the ileal alteration have been described. In the handful of cases in which the ileal cytomegalovirus lesions have been described, or in which illustrations of these lesions have been included, deep mucous ulcerations are usually reported $(2,5)$. However, these are very different from the ones which we present here, which are minute aphthas similar to those that occur in Crohn's disease, against which a differential diagnosis needs to be made.

\section{J.J. Sebastián, R. Uribarrena}

Endoscopy Unit, Gastroenterology Dept., Miguel Servet Hospital, Zaragoza, Spain

\section{References}

1. Smith P. Infectious diarrheas in patients with AIDS. Gastroenterol Clin 1993; 22: $535-48$.

2. Lepinski SM, Hamilton JW. Isolated cytomegalovirus. Gastroenterology 1990; 98: 1704-6.

3. Gonzáles Anglada MI, Barbado FJ, Arribas JR, et al. Terminal ileitis due to cryptosporidium and cytomegalovirus in a patient with AIDS. An Med Interna 1994; 11: 139-41.

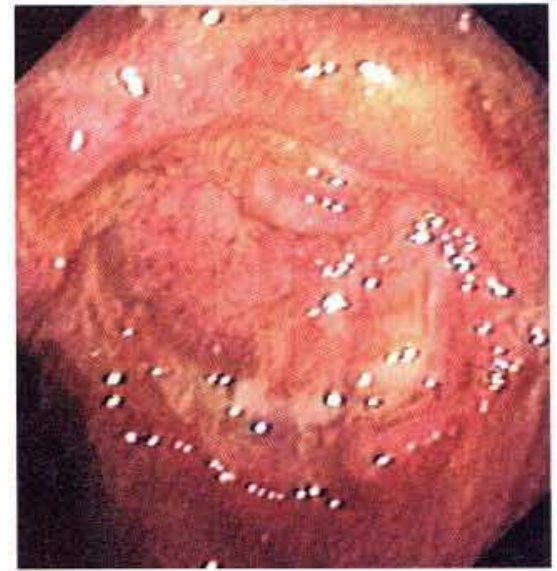

Figure 1: Giant crater-like ulceration in the left colon.

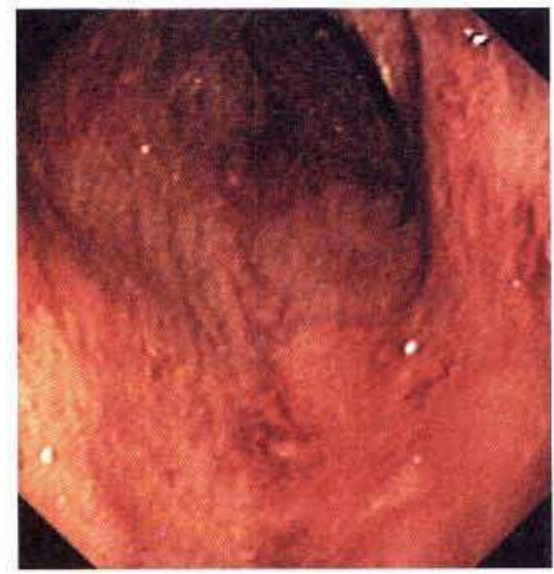

Figure 2: Minute aphthous ulceration and areas of submucous hemorrhage in the terminal ileum.

4. Gertler SL, Pressman J, Price P, et al. Gastrointestinal cytomegalovirus infection in a homosexual man with severe acquired immunodeficiency syndrome. Gastroenterology $1983 ; 85: 1403-6$.

5. Wajsman R, Cappell MS, Biempica L, et al. Terminal ileitis associated with cytomegalovirus and the acquired immunedeficiency syndrome. Am J Gastroenterol 1989: 84: 790 -3.

Corresponding Author

J. J. Sebastián

Endoscopy Unit

Gastroenterology Dept.

Miguel Servet Hospital

Paseo Isabel La Católica 1-3

50009 Zaragoza

Spain 